\title{
Representation of on-line questionnaires in an editable, auditable database
}

\author{
HOWARD L. KAPLAN \\ Addiction Research Foundation of Ontario, Toronto, Ontario, Canada
}

\begin{abstract}
A general way of describing multiple-choice questionnaires for on-line presentation is described. Some of the important features supported include random ordering of and sampling among questions, substitution of specific-question text into boilerplate question and response formats, a limited ability to bypass irrelevant questions, experimenter editing of response arrays, and automatic production of hard-copy versions of the questionnaire for use during computer breakdowns and for archival documentation. All editing operations on both questionnaires and response data create audit trails for future reference.
\end{abstract}

One of the oldest uses for computers in psychology is the automation of the presentation and scoring of multiplechoice questionnaires, especially of the type used in clinical settings. (Similar questionnaires used in educational applications are beyond the scope of this paper.) The programming of a simple questionnaire may be a straightforward exercise in a language such as Pascal or BASIC: an inner loop that displays responses is nested within an outer loop that displays questions, and consecutive responses are saved in a simple vector. The implementation questions become more interesting, though, when one wants to efficiently present, score, and document a variety of questionnaires, especially when some violate the simple computer structures of nested loops and singly subscripted vectors.

As part of a general redesign of software for measuring psychopharmacological effects on behavior, I am currently implementing a general form-based tool for defining, presenting and scoring, and documenting the classes of questionnaire most useful to us. Since very little of what has been presented at this conference or previously published in Behavior Research Methods, Instruments, \& Computers (BRMIC) discusses the data structures used to implement such software, that will be one of the major emphases of this paper.

\section{Previous Reports}

None of the first three conferences of the Society for Computers in Psychology (1971-1973) featured any papers specifically devoted to the subject of automating multiple-choice tests. Two papers presented in 1974 (Cole, Johnson, \& Williams, 1975; Space, 1975) discuss implementation issues that are still relevant today: What is the appropriate response device, especially for use with subjects not skilled in using a standard computer keyboard? What kinds of analyses should be attempted on-

Correspondence should be addressed to H. L. Kaplan, Addiction Research Foundation, 33 Russell St., Toronto, Ontario M5S 2S1, Canada. line, and what kinds should be deferred for later computation? How can one ensure that the structure of each test is documented in a form more generally understood than a computer programming language? How should one divide the work of computerizing several different tests into modules that allow the maximum efficiency in reusing program code?

In subsequent years, presentations were less concerned with issues of implementation technology. A six-paper session was presented the following year. One of those papers (Johnson, Giannetti, \& Williams, 1976) was a review of 15 years' history of the use of computers in mental health care delivery. While most of these six papers emphasize the psychiatric utility of on-line assessment, only one of them (DeWitt \& Weiss, 1976) discusses some of the technical issues involved in actually implementing such a system. In 1976, six papers were again presented and, again, the emphasis was on such issues as validity and professional acceptance of the scoring. In each of 1977 and 1978, there was one paper on questionnaires as part of a general session on clinical applications; however, in 1978, there was also a session featuring four reports from a group at the University of Rochester about a microprocessorcontrolled psychopathology laboratory, with the paper by Huntzinger and Space (1979) devoted to software issues in questionnaire development. Their report emphasized standard data formats and modular libraries of routines available to programmers; construction of new tests still apparently required at least a beginner's familiarity with a programming language to call such routines. No relevant papers were presented in 1979.

In 1980, a paper by 14 authors (Johnson, Harris, et al., 1981) only briefly discussed implementation issues (e.g., whether one should require confirmation of responses or allow backtracking after self-recognized errors), concentrating instead on descriptions of new applications. No relevant papers were presented in 1981, 1982, or 1983. In 1984, a paper by Perlman (1985) about the automatic generation of "surveys" can be read as describing a language for defining and presenting a class of questions in- 
cluding, but not limited to, multiple-choice questions. There was another hiatus until 1989 , when there was a paper by Klieger (1990) on a system for automating paperand-pencil tests. Like Perlman's system, Klieger's was designed for use by nonprogrammers; it is limited to truefalse responses but can provide for scale scoring based on the questions that the branching logic never bypasses.

Outside of this conference, all of the papers published in BRMIC since 1977 (the starting date of the Computer Technology index published in 1987) that are relevant to questionnaire presentation come from two special theme issues. The August 1978 special issue "Microprocessors and Microcomputers" included a section of three articles on clinical testing and assessment. Johnson and Williams (1978) reported the successful redevelopment of software for a microcomputer; they state that the programs were written in BASIC and heavily overlaid, but they discuss only the data representation of the responses, not of the questionnaires. Bremser and Davidson (1978) also reported that their questionnaires were developed in BASIC, but they did not state how the questionnaires are represented in the computer.

Of the 35 papers in the August 1981 BRMIC special issue "Computer Technology and Methodology in Clinical Psychology, Psychiatry, and Behavioral Medicine," three are of particular relevance to this paper. Johnson and Johnson (1981) and Johnson, Godin, and Bloomquist (1981) discuss some of the human-factors issues in administering tests on line. Vale (1981) provides an extensive description of a test-specification control language in the context of adaptive ability testing (i.e., selection of optimally relevant test items based on responses to earlier presented ones). The language so described was prepared as a text file and compiled by a batch process, resulting in a compact testing module for quick execution. The language refers to questions only by number (e.g., "INSO01"); the question text is stored under that number on a separate ASCII file and combined with the control logic during the batch process.

In summary, the automation of multiple-choice questionnaires seems not to have generated many technical reports analogous to those reporting the automation of laboratory procedures, such as operant conditioning or cognitive testing. In particular, there have been very few attempts to put tools for questionnaire development into the hands of nonprogrammers. In this report, I present some technical information that may assist the work of other developers in this area.

\section{Our Development Context}

One area of research at the Addiction Research Foundation is behavioral pharmacology. Since 1984, some of this work has been conducted under versions of the Scheduled Measurement System, or SMS, on which I have reported previously (Kaplan, 1985, 1987; Kaplan \& Noldy, 1989). Briefly, SMS provides an MS-DOS computer with a framework of scheduling, data management, and $\mathrm{I} / \mathrm{O}$ control routines under which a variety of measurement modules execute. These modules can include performance measures (e.g., psychomotor tracking or memory), physiological measures (e.g., evoked potentials), and subjective measures assessed by checklists and questionnaires. We are typically measuring effects that change over the course of hours, and, therefore, we repeat a cycle of tests five to 10 times per day; the entire daily procedure is then repeated for different drug conditions in a crossover design.

Our current use of SMS is largely to assess abuse liability, the likelihood that a drug will be taken for recreational purposes. Briefly, to assess this, we compare subjects' responses to test drugs with their responses to placebo and to drugs of known abuse liability. Because some of this work is performed under contract to pharmaceutical manufacturers seeking regulatory approval for marketing new drugs, it is necessary for us to provide complete written descriptions of our proposed measures in research protocols, document the measures actually used after our designs have had a meaningful encounter with reality, and demonstrate to outside auditors that the data reported are the same as those actually collected.

As I reported 5 years ago (Kaplan, 1987), SMS worked better as an aid to the programmer implementing an experiment than it did as an aid to the research assistant trying to conduct it or to the senior researcher trying to meet the requirements of regulatory agencies. Even for a programmer, though, it had serious deficiencies: being programmed in Turbo Pascal Version 3, it was difficult to debug, and language limitations did not conveniently support the maintenance of such large programs. In addition, too many parameters were hard coded into the programs rather than being placed in easily editable tables, necessitating frequent recompilations for trivial changes. In addition, the only hard-copy documents automatically produced by the system were printouts of daily data and data dictionaries for all measures. These dictionaries included the abbreviated names (but not the actual text) of all questions, the range of values that each response or summary scale might span, and the columns on the output record holding the data. This kind of data dictionary is useful in assisting a data analyst already familiar with an instrument in reading the data files, but not in explaining the instrument to a new audience.

As technology has improved, the originally imposed design limitations (that it run well on $256 \mathrm{~K}$ memory computers without hard disks) and goals of SMS have become outdated, and the temptation to redevelop it using modern compilers and more realistic restrictions finally became irresistible. One part of that redevelopment has been a rethinking of the way we organize questionnaires.

Our earliest SMS questionnaires were each separate programs that included an array of questions declared as a Turbo Pascal structured constant. One part of the program would loop through these questions when it was time to present them on the screen; a second part would loop through them to construct the data dictionary. This method was used to develop computer-administered versions of the Profile of Mood States (POMS; see Johansen \& Uhlen- 
huth, 1980) and the Addiction Research Center Inventory (ARCI; see Haertzen, 1974; see also Cole, Orzack, Beake, Bird, \& Bar-Jal, 1982). Both of these questionnaires are characterized by having a consistent response set across all questions and by having predefined scoring scales.

We subsequently needed to administer several different batteries of individual items, not intended for compilation into scales, where the response structure was not necessarily uniform across the items. For example, one item might ask the subject to compare a test drug with marijuana, with responses being "I prefer this drug," "I have no preference," "I prefer marijuana," and "I have never tried marijuana"; another item might ask whether a test drug's effects were "increasing," "holding steady," or "decreasing." For these questionnaires, we developed a better way to separate code from data and to avoid writing new programs. The questions and responses were simply edited as an ASCII text file, and that file was loaded into appropriate data structures at the time the questionnaire was to be administered. Special codes within the text indicated the key words that were to be included in or excluded from the data dictionary: for example, although the screen could display a question of well over 100 characters, we needed a 40-character, abbreviated version to use as an SPSS or SAS variable label.

Having the questions as an external text file had both advantages and disadvantages. Because the file could be edited between sessions of an experiment, it was possible to correct (or to introduce) typing errors or poor wording; however, there was no automated way to audit the history of such changes or to verify that the changes did not alter the data validity or storage formats. Also, the questionnaire interpreter required a strict syntax for question and response text but crashed rather than providing diagnostic messages when the syntax was violated. It became clear to us that the process of entering and validating questions and responses needed more computer assistance.

These experiences led to the following design goals for the redevelopment of questionnaire administration under the latest version, SMS 4:

- To allow the development and execution of questionnaires to be tolerable on computers as slow as $8-\mathrm{MHz}$ $\mathrm{XT}$ clones with $640 \mathrm{~K}$ of memory and a hard disk drive.

- To allow new questionnaires of a variety of structures to be implemented by staff with computer skills similar to those required for word processing.

- To provide developers with immediate feedback about the questionnaires under development, both by providing error messages and by demonstrating the execution and scoring of the questionnaire.

- To allow the automatic substitution of question-specific text into boilerplate question and response text.

- To allow blocks of questions to be presented in random order, to be randomly sampled from, or to be presented only when earlier questions have specified responses.

- To restrict the kinds of changes made after an experiment begins to those that do not alter the structure of the data file, and to maintain an audit trail of all such allowable changes.

- To continue the practice of presenting questions and responses in a highly legible text about twice as large as the usual text mode ( 25 rows $\times 80$ columns) characters.

- To continue the practices of automatically constructing a machine-readable data dictionary and of using that dictionary to allow experimenter editing of completed data records, but to add an audit trail of any such editing operations.

- To produce documentation files about each questionnaire from the data used to control its real-time presentation, such as files easily importable into a word processor for minor formatting before printing.

\section{The Development Environment}

There are two levels of development involved here. I work at the level of developing code in Turbo Pascal Version 6 to create the supervisory program SMS 4 and a questionnaire-specific program, QCONTROL; users of that combination develop questionnaires. My software work environment includes the Turbo Pascal compiler and debuggers (products of Borland International), the subroutine library Turbo Professional (a product of Turbo Power Tools), and a number of general-purpose subroutines of my own design. Some of the most important of these home-built routines were described last year (Kaplan, 1991): a singly indexed random-access file manager, used here to store questions, responses, scale names, and so on; and a table-driven screen manager that allows the presentation and editing of structured records without the need for me to explicitly code any dialogues or paint any screen forms.

In earlier versions of SMS, the measurement modules were very tightly coupled to the supervisory control program; modules were literally program overlays sharing data and subroutines with the supervisor. In SMS 4, where computer memory is sufficient to allow for greater redundancy, the coupling is much looser. Only a limited amount of information is passed between SMS 4 and QCONTROL, primarily these items: the questionnaire name, an appropriate seed for the random number generator, a one-character parameter that selects boilerplate introductory question text and controls the skipping of certain questions, the address for storing the response vector, and the address for storing a copy of the data dictionary that can be used to edit or print results. This loose coupling reduces the size of the code that I need to develop and debug at any one time, an important factor given the limited memory available when using the Turbo Pascal integrated debugging environment; it also allows for the subsequent development of other kinds of measures that are not questionnaires.

\section{Questionnaire Data Representation}

Five data types are sufficient for describing any questionnaire that can be developed under QCONTROL: title, 
header, scale, response, and question records. There is exactly one title record, but the other record sets consist of a variable number of records. Each such record is indexed by the name of the questionnaire to which it belongs, and it also contains fields (e.g., "Question set name" or "Response set name") to allow specific records to be referenced by other records. The resulting database is not a relational database in the formal sense, but its design has been influenced by that of true relational databases. The records of this database are edited by the developer using simple screen forms, which are illustrated here using portions of the ARCI questionnaire.

The title record (Figure 1) contains versions of the title suitable for including on printed reports and on the screen (the latter divided into shorter lines), the stub (e.g., "ARCI"), which gets expanded into variable names (e.g., "ARCI001" through "ARCI166") when the data items are exported for statistical analysis, and the physical arrangement of responses on the screen.

The header records (Figure 2) are optional. Each one consists of a control character and of boilerplate text that prefixes every question when the control character matches the input parameter passed by SMS.

The scale records (Figure 3) are optional. Each one defines the title for a summary scale, gives it an identifying letter or number for reference on the question records, controls whether a negative coefficient means that the response value is subtracted or that the assignment of scores to responses is reversed, and controls whether the summary scale is normally printed and graphed (the score is always stored on the database even if hard-copy output is inhibited). The scoring coefficients are stored as part of the question records, described below.

The response records (Figure 4) are mandatory. If there is only one response set applicable to the entire questionnaire, then it need not be given a name; otherwise, each response set is named separately. Each record includes the response-set name, the response-set scale type (nominal, ordinal, or interval), ${ }^{1}$ the response numeric value stored on the data file, the response text shown on the screen, and the abbreviated response text stored in the data dictionary as a 16-character value label (if that text is different).

The question records (Figure 5) are mandatory. Questions must occur in named sets, and one of these sets must be named "MAIN"; other sets are included in the presentation only when referenced by a question that is part of the MAIN set. Each record includes the question-set name, the question text presented on the screen, the question text in the form of a 40-character variable label (if different), the response set to be used, the percentage of the referenced questions to be included, the condition under which the nested questions are to be included, the question's weight on each of the scales defined, and whether or not the question is included on the hard-copy daily printouts and graphs. The nesting logic by which questions reference other question sets is described in more detail below.
As the developer edits each of these record types, QCONTROL checks for the independent validity of each field and the consistency of fields within and between records of any one type. For example, if a response scale is said to be interval, then the response numeric values must be uniformly spaced. Some consistency checks, however, require the inspection of both the question and the response data sets: for example, all response sets referred to by the questions must exist among the responses. Therefore, QCONTROL requires any sequence of editing operations to end with an editing of the questions. If the questions were not the last type edited, then only a limited menu of editing options is available; after they are edited (and their consistency with the scales and responses are verified), a larger menu allows committing the changes to disk and testing the resulting design (Figure 6).

The code for auditing the changes to the questionnaire is still under development. As currently designed, one of the fields maintained on the title record contains the date and time when the questionnaire was "locked" (i.e., when the pilot study terminated and formal data collection began). Only selected modifications to the questionnaire (described below) are allowed after such locking; the person making the modifications must also complete a form describing the nature of the modification and the reasons for it. This audit trail becomes a permanent part of the questionnaire database and is printed on the document describing the questionnaire. An automatic checksum built into the file containing the questionnaire structure can be used to verify that no changes to the file have been made except through this auditable process.

The only changes allowed to a questionnaire's contents once it has been locked must be those that do not violate the comparability of the data before and after the change. Program logic can automatically disallow some changes, but the validity of others can be determined only by the experimenters. Some changes, such as whether or not a particular summary scale is graphed on the daily printout, have no effect on data validity and do not require an explanation in the audit trail. Other changes, such as altering a word within the text of a question, could be either a simple correction of a spelling error or a substitution that totally alters the meaning of the item. Since the computer cannot distinguish between these alternatives, it records all wording changes in the audit trail. Changes that alter the structure of the data-storage records, such as adding a new question between two existing ones or before the summary scales, are always disallowed in a locked questionnaire.

\section{Question Nesting}

There are often circumstances when one wishes to enter a list of partial questions (e.g., adjectives) once and present the list in several different contexts during the presentation of a questionnaire. For example, one may need to have a subject rate both current mood and expectation of mood over the next hour using the same adjectives, or one might want to readminister $10 \%$ of a questionnaire 
Edit the title information

8-letter identifying name Consarci

Questionnaire title on reports Addiction Research Centre Inventory

Questionnaire title on screen Addiction/Research/Center/Inventory

Common stub of all variable names ARCI

Response display format Vertical

F1:Help

F 7: $\uparrow$

9: 1

Figure 1. The editing screen for the title records.

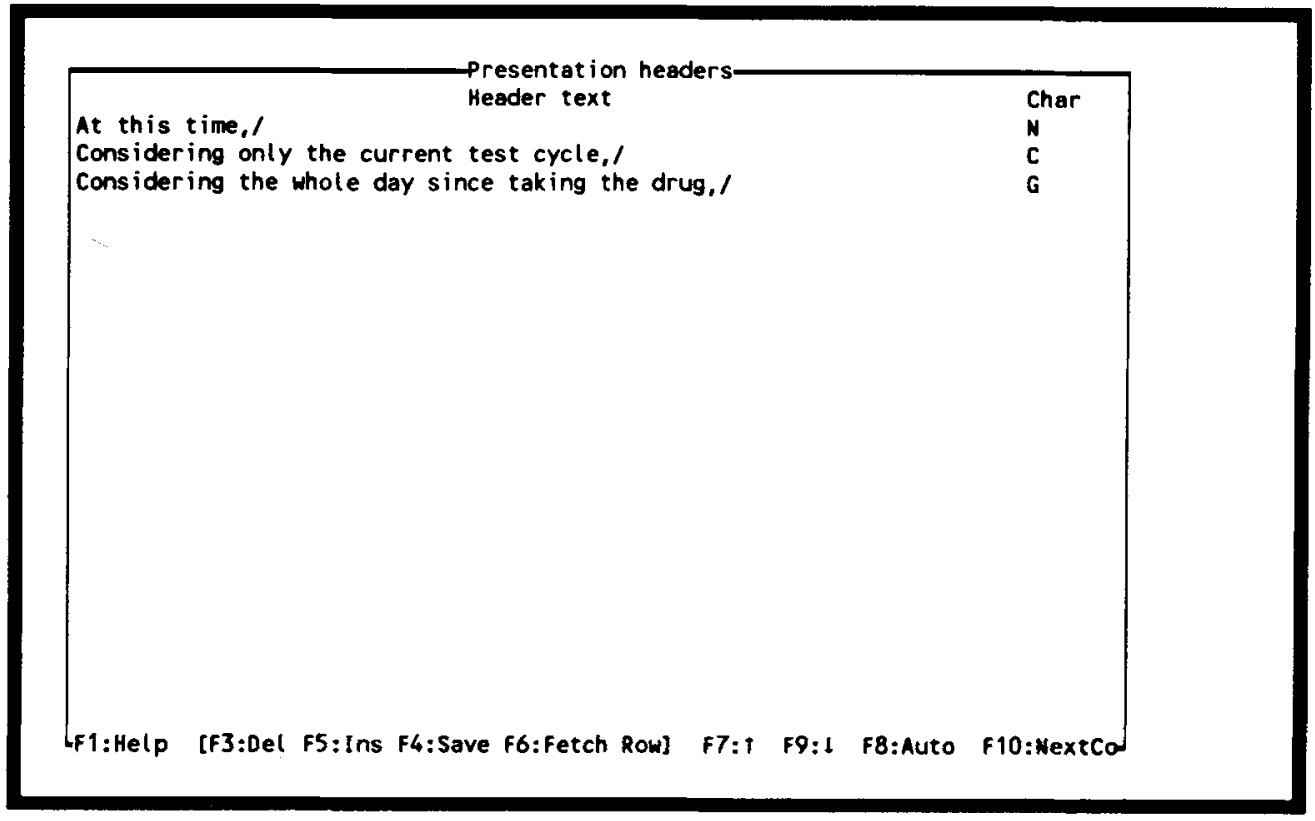

Figure 2. The editing screen for the header records. 


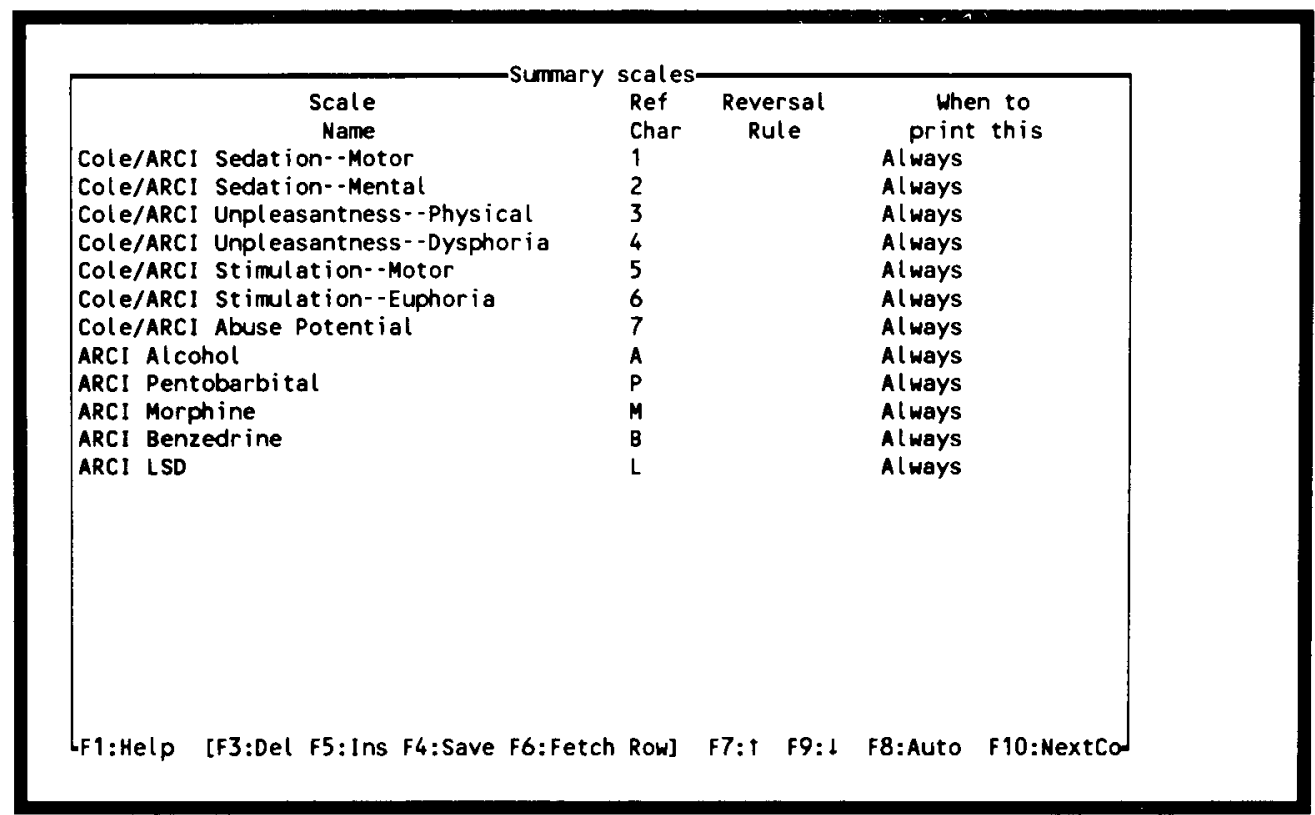

Figure 3. The editing screen for the scale records.

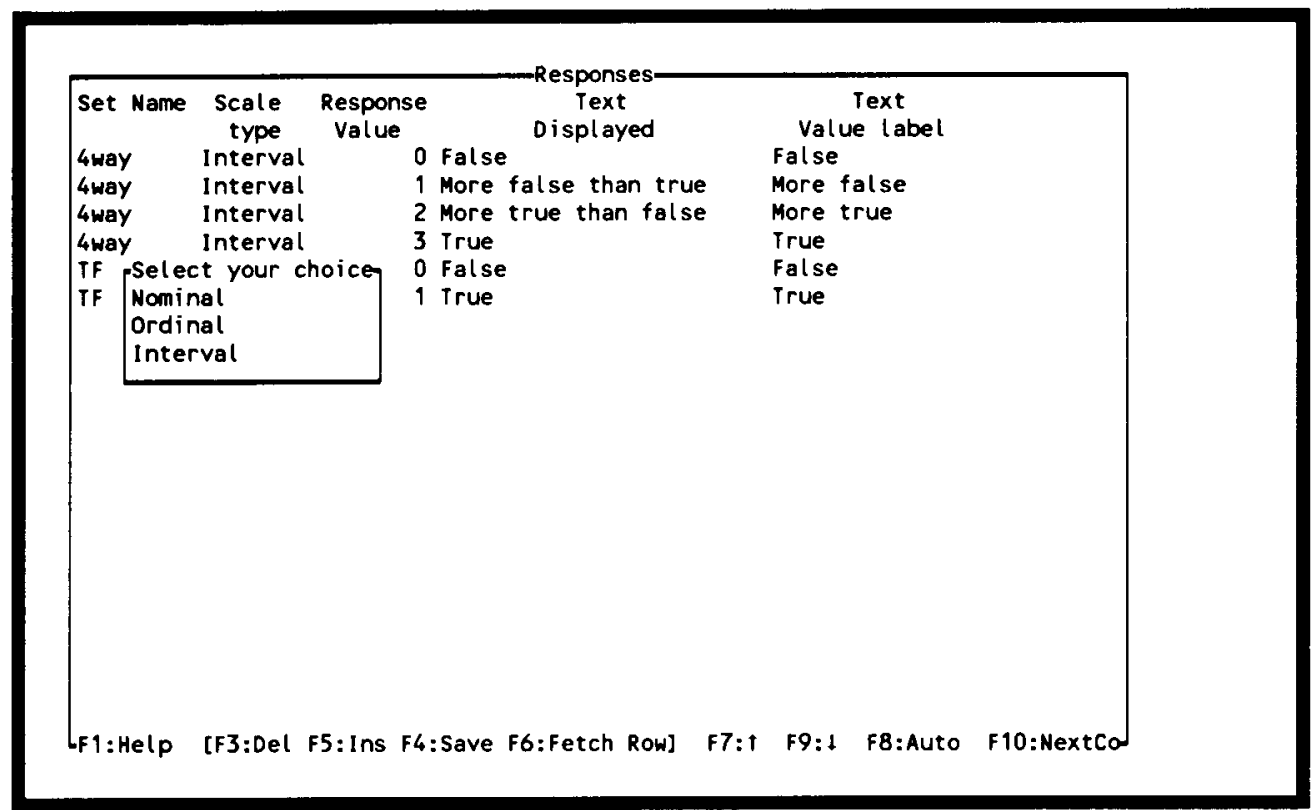

Figure 4. The editing screen for the response records. Note the small sub-window displaying the possible choices for a field that allows only a limited set of alternatives. 
(A)

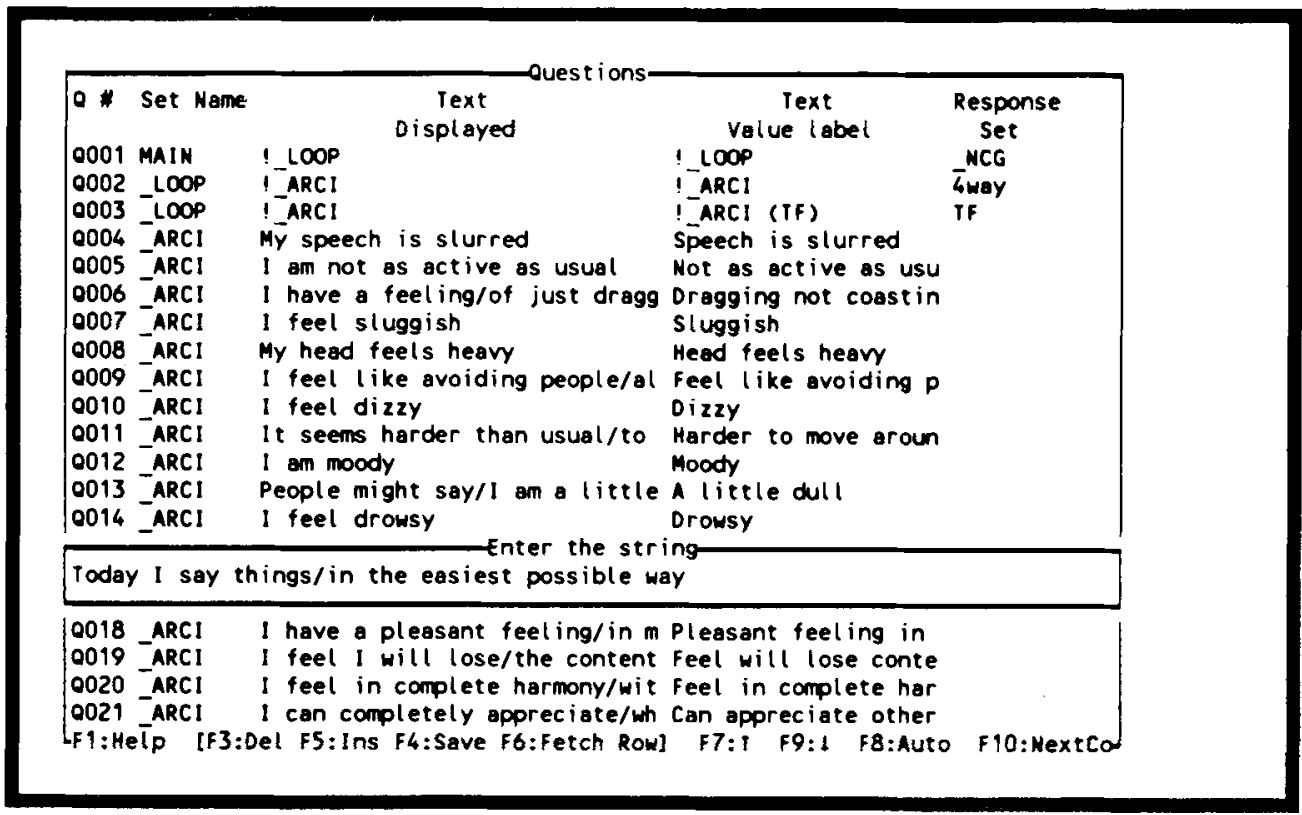

(B)

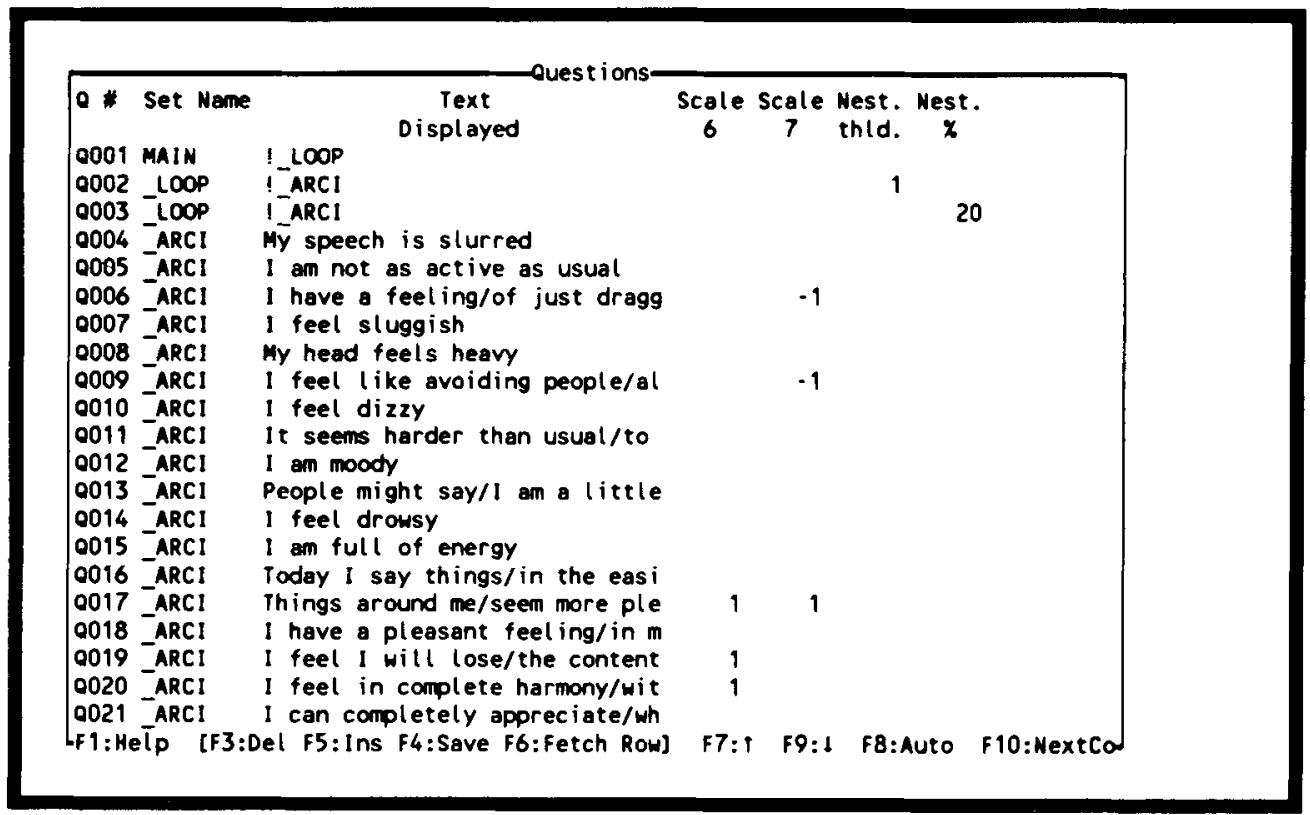

Figure 5. (A) The leftmost portion of the editing screen for the question records, showing a field expanded beyond its normal display width during editing. (B) Another portion of the screen for editing questions, showing the area where the question weights on the summary scales can be entered. 


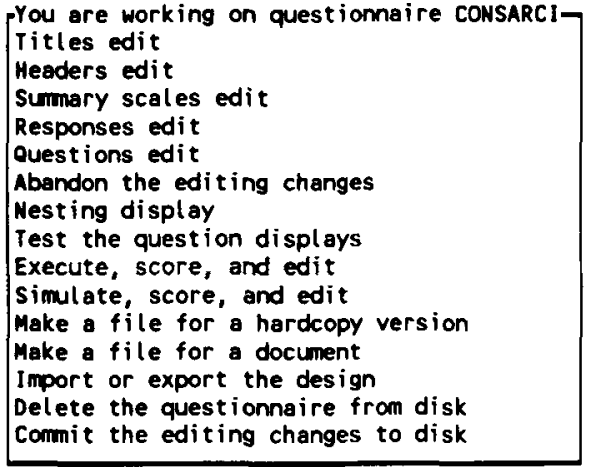

Figure 6. The full set of editing options available after the questions have been checked for consistency with the responses; before that time, only the first six lines of the menu are available.

as a consistency check or as a test of a novel phrasing of the response scale. One might also need to entirely eliminate the presentation of a set of questions irrelevant to the presentation circumstances. These needs can all be met through the use of nested questions and appropriate auxiliary control fields.

In its simplest form, question nesting occurs when the text of a nesting question includes an exclamation point followed by the name of a question set (e.g., "I am feeling !AdjSet1"). The inclusion of this question is an abbreviated representation of a number of individual nested questions: for example, "I am feeling annoyed" and "I am feeling tense," where "annoyed" and "tense" are display text entries in the nested question set AdjSet1. Also, the text of the lowest level of nesting can be substituted into the response text: if the latter contains an exclamation point, then question text is substituted into the response at that point (e.g., the response "I prefer !" may be displayed as "I prefer marijuana" or "I prefer amphetamine").

More complex control over nested questions is also available. The questions within each set at the lowest level of nesting can be randomly sampled, and one or more levels of nesting can be bypassed entirely. Within the question definitions, any nesting question can specify a number between 1 and 100 as the percentage of nested questions to include; if the number is not specified, all are included. If a question specifies a response set whose name begins with an underline character (e.g., "_BD"), then the response is not sought from the subject; instead, the onecharacter parameter passed by SMS is inspected. If that character is one of the letters in the response-set name, then it is considered to be implicitly answered "yes." For example, if a questionnaire includes some questions relevant to an entire day and others relevant only after drug or placebo has been given, then SMS can pass " $B$ " for baseline during the predrug test cycles and " $D$ " for drug during the postdrug ones. In that case, a question marked "_BD" would be deemed to have been answered "yes" in both circumstances, whereas one marked "DD" would be so deemed only in the postdrug condition.

Either a question answered automatically by reference to the passed parameter or one answered by the subject may control the presentation of subsequent questions. It may be followed immediately by a nesting question that tests its predecessor's response and includes nested questions only if the tested response meets a specified Boolean condition. For example, after the question, "Did you know your birth mother after you were twelve years old?" the subsequent nesting question could have the text, "Did your mother ever feel !AdjSet1?"' and have the condition that the previous response be 1 (as a numeric value for "yes"), thereby implementing an assessment of the mother's range of mood states only when that is relevant.

The same questionnaire control parameter that can be used to bypass irrelevant questions also controls the introductory text displayed above all questions. This is useful, for example, when one wants to have several administrations during the day of questions that begin "Right now, I am feeling ...," followed at day's end by a summary rating of questions beginning "During the day, I usually felt ..."

In addition to passing a questionnaire control parameter, SMS passes a randomization seed. Within a questionnaire, questions at the lowest level of nesting are always 
presented in a random order, constrained so that all subjects at the same phase of an experiment (e.g., Test Cycle 1 of Day 6) receive the same randomization. (In our usual Latin square designs, days are orthogonal to drugs, so the randomization of questions would also be orthogonal to drugs.)

Whenever a set of questions is $100 \%$ sampled (although it may be bypassed when irrelevant), summary scales can be scored for that set of questions. When there are multiple scales, then scale weights are assigned to individual questions (a question may weight on more than one scale); when there is only one scale on which all questions weight equally, then that weight may be specified on the nesting question instead. To distinguish among scales when the same nested questions are nested under several different nesting questions, response-scale names on the data dictionary incorporate question-set names between "MAIN" and the lowest level of nesting. For example, a POMS scale simply called "Tension-Anxiety" on the scale records may appear in the data dictionary as both "Current Tension-Anxiety" and "Usual Tension-Anxiety," on the basis of the same adjectives presented in different surrounding text.

\section{Run-Time Control}

Questions are presented in monochrome in $640 \times 200$ pixel CGA or $720 \times 348$ pixel Hercules graphics mode. Question and response texts are presented in a font approximately twice as large as the default, 25 rows of 80 characters text font; a smaller font is used for the option of returning to the previous question. A provision to eventually manage four response-selection devices is included, even though we are now using only the first two: a light pen, the standard keyboard, a mouse, and a joystick.

A light pen (normally available only for CGA or EGA monitors) is in many ways the most natural device: the subject simply uses the pen to touch a block-shaped target adjacent to the preferred response. However, some subjects find the use of light pens tiring despite our provision of elbow rests, and the pens occasionally return incorrect screen coordinates. To reduce the likelihood of such incorrect coordinates being taken as correct, the software requires confirmation of the coordinates on two consecutive screen refreshes. To reduce the likelihood that such an incorrect choice, if taken as correct by the computer, will go unnoticed by the subject, we reverse the colors of the entire response area after a response is chosen via any of the four devices (Figure 7), so that the subject can, if required, go back to reanswer the question. A touch screen, which we have not implemented, would provide many of the same advantages as does a light pen.

We use a keyboard as the response device only during development or demonstrations, since responding is not straightforward. Because we are not normally displaying any letters or digits alongside the words that comprise the possible responses, the developer cannot press a correspondingly labeled key to select a response. Instead, the developer uses only three keys: The " $>$ " and " $<$ " keys

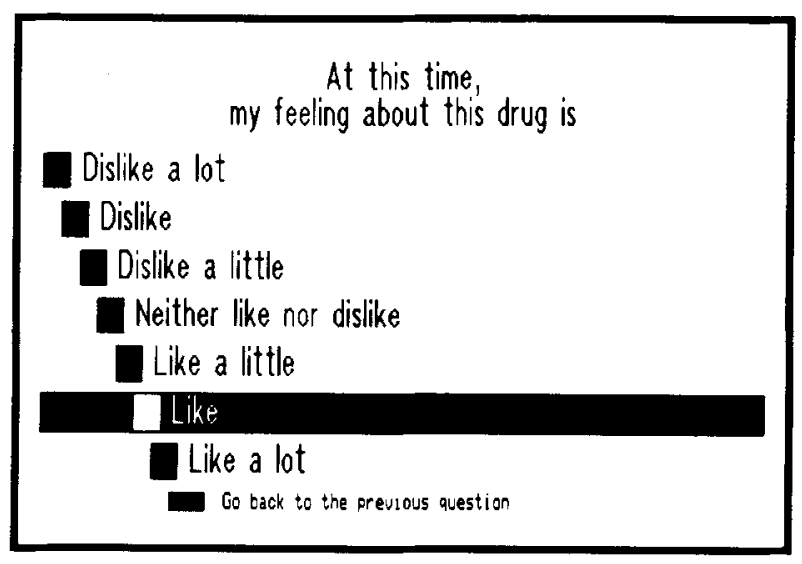

Figure 7. A typical display of a question and its responses, shown at the point where the subject has just selected the sixth alternative, which is highlighted for feedback. Although shown here as dark text on a bright background, questions are actually displayed as bright text on a dark background.

(whether or not shifted) move an arrow cursor down or up the response list, and the space bar selects the response currently indicated by the arrow.

In the past, we have been reluctant to ask sedated subjects to use a mouse to point to response selections, fearing that such subjects would not have the proper motor control for such a device. However, we are including the option in SMS 4, and we will conduct some empirical tests of accuracy and latency if we decide to offer it as an alternative to the light pen. We do not normally collect latency measures, but it is clear to us that a pointing device with an excessively long latency can frustrate the subject and lead to less accurate responses. We have also tried using a joystick in a mouse-like mode, controlling an oval cursor that ranges over the screen like a mouse cursor, and we have found that we need to impose a lowpass digital filter (a simple smoothing function) on the joystick position to reduce instability in the position of the cursor. Our prediction is that the difficulty of controlling the position of the oval joystick cursor, despite the filtering, makes the current light pen software better than any possible joystick-based software, perhaps because of the limited resolution of joysticks and their interfaces. However, we are aware of a higher precision, joystick-shaped mouse substitute called the "ICONtroller" (Suncom Technologies); this may be worth further investigation.

Regardless of the response device, subjects may realize that the recorded response (as indicated by the screen color reversal) is not the response they intended to give. Therefore, all response lists include an option, in smaller type at the screen bottom, to go back to the previous question. Because the questions presented on any one occasion depend on the randomization and possibly on responses to earlier questions, the backtracking pointers are constructed at run time as each question is presented. After all substantive questions of a questionnaire are presented, a final 
screen is always automatically included, giving the subject the opportunity to backtrack to the last question presented.

\section{Data Management}

Corresponding to every questionnaire is a data dictionary that includes one record for every question that would be presented if none were bypassed at run time, along with one record for each summary scale computed. Such summary scales are based only on the lowest level nesting groups for which the sampling proportion is $100 \%$, on the assumption that the purpose of fractional sampling is not to produce summary scales but instead to validate randomly selected individual questions.

In the data dictionary, the eight-character variable names (suitable for use in standard statistical packages) terminate in consecutive numbers (e.g., "POMSO1" through "POMS82' could refer to the standard 72 POMS adjectives followed by the standard 10 summary scales). The 40-character variable labels are specified on the questions form, with the same kind of substitutions for nested questions allowed in the variable-names text as are allowed in the screen display text. As described earlier, the variable labels for the summary scales are constructed of a concatenation of question-set names and scale names. Other data dictionary fields include the ranges of valid response values and the text fields associated with the response values. The dictionary can be used for the automatic production of SPSS or SAS text to build data files or for the manual editing of responses.

To allow research assistants to edit individual responses that the subjects report as errors but do not go back to correct, as well as to enter responses made on paper ver- sions of the forms used if the computer malfunctions, an editing subprogram uses the data dictionary to present responses as a scrolling screen form displaying 21 questions' labels and responses at any one time (Figure 8). The summary scales are also shown on this form but are marked as nonalterable items; only the individual responses may be changed by the editing process. When the changes are accepted, the person who performed the edit is presented an additional screen for describing the reason why manual changes were made (Figure 9). When that is complete, the summary scales are rescored, and both the revised data vector and the reasons for the changes are stored on disk. The date of such changes and the reasons for them are included in the printed version of the day's data, in order to provide an audit trail for future validation of the data. A checksum similar to that used on the questionnaire file itself can be used to validate the audit trail.

The standard printout at the end of a day is organized by test. Tabular data is printed as one column per cycle, and graphed data is printed over an axis whose abscissa is the elapsed time relative to time zero, the time of the drug administration. Not all of the data collected need be printed in detail. When validated summary scales exist, it is usually just those scales, rather than the individual questions' responses, that are printed and graphed. The scale and question data entry forms allow the research staff to specify which of these items are to be printed and graphed, and under what circumstances. An item can be printed always, never, or only if there is any change during the day; an item can be graphed using either an ordinate that spans the entire range of valid values or just

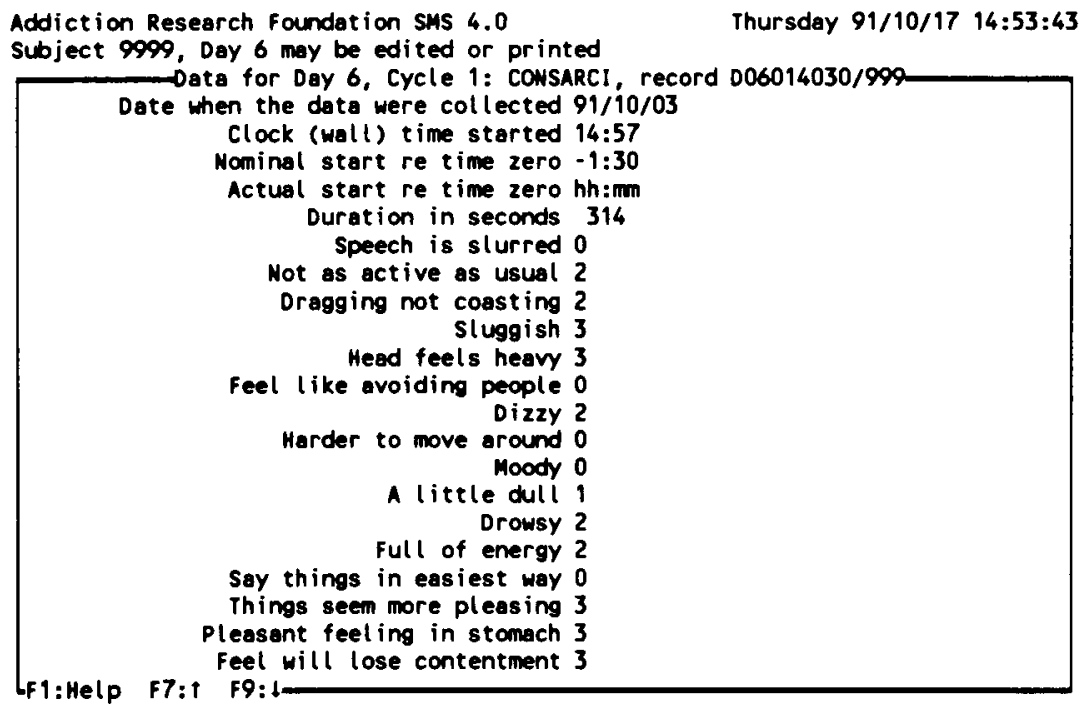




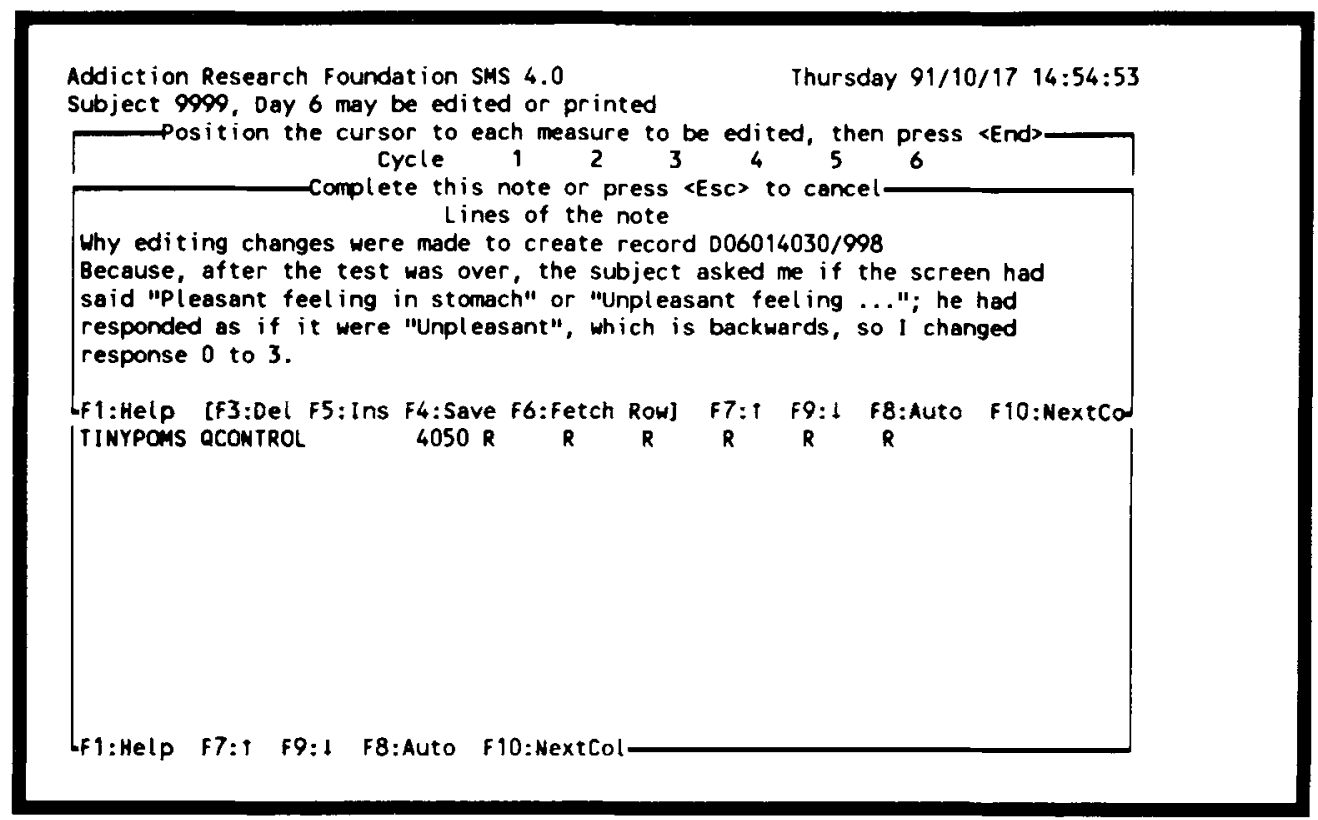

Figure 9. The editing screen that the research assistant must complete to explain why data were manually altered after the subject completed a questionnaire.

the range required to show the values actually achieved. For individual questions, the ordinate is marked with abbreviated response labels rather than numeric labels. Printout can be directed to either inexpensive, nine-pin dotmatrix printers or to faster LaserJet-compatible printers.

All data from a study, including questionnaires and other measures, are stored on a single file per subject. This file is normally stored in a compressed form on backup diskette but in an expanded, rapid-access form on the hard disk during the hours when a study is running. Having each subject's compressed file on a separate diskette facilitates moving subjects from computer to computer on different testing days, as we have a non-networked environment where several subjects can be tested on a single day.

\section{Printed Documentation}

There are two reasons for reducing an on-line questionnaire to a printed form. Much of the paperwork surrounding our studies is still, literally, paperwork: pharmaceutical companies and regulatory agencies want to have printed documents about the measures used in a study. We therefore need to have a professional-looking document about each measure, explaining exactly how it is administered and how the data are interpreted. This is necessary even when we are simply computerizing a published instrument, so that any project staff not familiar with the instrument can see exactly what questions and responses are included. Therefore, one form of printed output contains all questions (including the appropriate expansion of nested questions), including notes such as "Only 25\% of the questions in this section are presented" or "This section is presented only when the input parameter is ' $F$ '." In this form, response sets shared among many questions are usually described only once, at the beginning, rather than repeated under each question.

A separate need for a printed form occasionally arises from computer breakdowns or from the need to present a normally computerized form off-line to a large group of subjects. In those circumstances, we need a paper-andpencil version of the questionnaire that presents exactly the questions that would occur under one specific initialization of the random number generator, with all responses detailed under each question, and with instructions to skip to specific questions depending on the response to the previous question.

Almost all of the work of producing these two separate printed documents is performed by QCONTROL. The text for the documents is produced in "generic" wordprocessor format, with all paragraphs appearing as long lines and with no page breaks specified. Most word processors (for example, Word Perfect, Microsoft Word, or Textra) can easily import such a file, allow an operator to make minor modifications for appropriate page headers and page breaks, and output the result to a good quality printer.

\section{Development Resources}

Not including the source files in purchased libraries, the Pascal source files needed to build SMS 4 and QCONTROL currently occupy about $600 \mathrm{~K}$ of disk space, and the development is about $75 \%$ complete. Most debug- 
ging can be accomplished using the integrated Turbo Pascal debugger rather than the stand-alone one, but only when a 386-memory manager is used to extend the DOS conventional memory area from $640 \mathrm{~K}$ to $736 \mathrm{~K}$, making use of address space otherwise occupied by the VGA adapter.

\section{Concluding Remarks}

The material presented here is certainly not at the cutting edge of either test theory or computer science. Although object-oriented methods have entered the microcomputer mainstream in the last few years, the work reported here is not a significant showcase for their use; such methods were used when it was convenient to do so, but much of the code is written in a standard, procedural form. Instead, the work reported here has two attributes that may prove to be useful sources of inspiration for other laboratories. The first is the emphasis on security issues, issues that will become of increasing importance to those of us working in applied areas that come under the supervision of government regulatory agencies. Software that at least detects, if not prevents, unauthorized tampering with the data files and that provides a complete history of authorized modifications is an important component of such applied research. The second is an emphasis on moving information from program code, where only compilers and cross-reference utilities can act upon it, to structured data files where a range of utilities can act upon it. This emphasis is consistent with current trends in commercial and administrative uses of computers. Just as a customer order entry database enhances the integrity of a product distribution process by reducing the opportunity for redundant data representations to get out of phase, so does a questionnaire structure database help to ensure that the documents used to describe, substitute for, and analyze an on-line questionnaire stay in phase with the actual questionnaire being administered.

\section{REFERENCES}

Bremser, R. F., \& DAvidson, R. S. (1978). Microprocessor-assisted assessment in the clinical research laboratory. Behavior Research Methods \& Instrumentation, 10, 582-584.

Cole, E. B., Johnson, J. H., \& Williams, T. A. (1975). Design considerations for an on-line computer system for automated psychiatric assessment. Behavior Research Methods \& Instrumentation, 7, 195-198.

Cole, J. O., Orzack, M. H., Beake, B., Bird, M., Bar-Jal, Y. (1982). Assessment of the abuse liability of buspirone on recreational sedative users. Joumal of Clinical Psychiatry, 43(2), 69-74.

DEWITT, L. J., Weiss, D. J. (1976). Hardware and software evolution of an adaptive ability measurement system. Behavior Research Methods \& Instrumentation, 8, 104-107.
HAeRTzen, C. A. (Ed.) (1974). An overview of the Addiction Research Center Inventory (ARCI): An appendix and manual of scales. Rockville, MD: National Institute of Drug Abuse.

Huntzinger, R. S., SPACE, L. G. (1979). A microprocessor-based psychopathology laboratory: IV. Software design. Behavior Research Methods \& Instrumentation, 11, 253-256.

Johansen, C. E., Uhlenhuth, E. H. (1980). Drug preference and mood in humans: Diazepam. Psychopharmacology, 71, 269-273.

Johnson, J. H., Giannetti, R. A., Williams, T. A. (1976). Computers in mental health care delivery: A review of the evolution toward interventionally relevant on-line processing. Behavior Research Methods \& Instrumentation, 8, 83-91.

Johnson, J. H., Godin, S. W., \& Bloomouist, M. L. (1981). Human factors engineering in computerized mental health care delivery. Behavior Research Methods \& Instrumentation, 13, 425-429.

Johnson, J. H., Harris, W., Thompson, C., Marcus, S., Block, D., Novak, K., Yingst, N., Allert, A., Hobin, G., Byrnes, E., Strauch, D., Feldman, C., Niedner, D., \& Fink, A. (1981). New uses for the on-line computer medium in mental health care. Behavior Research Methods \& Instrumentation, 13, 243-250.

JoHNSON, J. H., \&oHNSON, K. N. (1981). Psychological considerations related to the development of computerized testing stations. Behavior Research Methods \& Instrumentation, 13, 421.424.

Johnson, J. H., \& Williams, T. A. (1978). Using a microcomputer for on-line psychiatric assessment. Behavior Research Methods \& Instrumentation, 10, 576-578.

KAPLAN, H. L. (1985). When do professional psychologists need professional programmers' tools? Behavior Research Methods, Instruments, \& Computers, 17, 546-550.

KaPLAN, H. L. (1987). Incorporating good programs into the larger laboratory context. Behavior Research Methods, Instruments, \& Computers, 19, 210-214.

KaPLAN, H. L. (1991). Maintaining a minimassive database of microdrinking measures. Behavior Research Methods, Instruments, \& Computers, 23, 225-228.

KaPlan, H. L., \& Noldy, N. (1989). A general framework for defining evoked potential paradigms. Behavior Research Methods, Instruments, \& Computers, 21, 285-293.

KLIEGER, D. M. (1990). Flexible testing without programming. Behavior Research Methods, Instruments, \& Computers, 22, 138-141.

Perlman, G. (1985). Electronic surveys. Behavior Research Methods, Instruments, \& Computers, 17, 203-205.

SPACE, L. G. (1975). A console for the interactive on-line administration of psychological tests. Behavior Research Methods \& Instrumentation, 7, 191-193.

VALE, C. D. (1981). Design and implementation of a microcomputerbased adaptive testing system. Behavior Research Methods \& Instrumentation, 13, 399-406.

\section{NOTE}

1. Those who have studied database theory will be familiar with terms like "third normal form." The normal forms are ways of controlling the redundancy among data records, ensuring that every important relationship is represented exactly once. The response records violate these normal forms, because the relationship between a response-set name and its scale type is repeated for each record of the set. However, in this application, it was more convenient to repeat the correspondence than to define a separate table for the sole purpose of recording it only once per response set. 